\title{
IMPLEMENTATION OF SMOKE-FREE HOMES IN POLAND
}

\section{DOROTA KALETA ${ }^{1}$, ADAM FRONCZAK ${ }^{1}$, BUKOLA USIDAME ${ }^{2}$, ELŻBIETA DZIANKOWSKA-ZABORSZCZYK ${ }^{3}$, TERESA MAKOWIEC-DĄBROWSKA ${ }^{4}$, and PIOTR WOJTYSIAK ${ }^{5}$}

\author{
${ }^{1}$ Medical University of Lodz, Łódź, Poland \\ Department of Preventive Medicine \\ ${ }^{2}$ University of Massachusetts, Boston, USA \\ Department of Public Policy \\ ${ }^{3}$ Medical University of Lodz, Łódź, Poland \\ Department of Social and Preventive Medicine \\ ${ }^{4}$ Nofer Institute of Occupational Medicine, Łódź, Poland \\ Department of Work Physiology and Ergonomics \\ ${ }^{5}$ County Office, Piotrków Trybunalski, Poland
}

\begin{abstract}
Objectives: Exposure to environmental tobacco smoke (ETS) constitutes a threat to the health of many people. In order to diminish ETS exposure, countries (including Poland) implemented legal restrictions of smoking in public places and worksites. Currently more attention is also paid to reduce overall and residential ETS exposure by voluntary smoke-free home policy adoption. The aim of current analysis was to evaluate the prevalence and determinants of implementing smoking bans at place of residence among economically active males and females in Poland. Material and Methods: Data from cross-sectional, household study - Global Adult Tobacco Survey (GATS 2009-2010) were analyzed. The logistic regression model was applied for appropriate calculations. Results: Out of 3696 studied subjects only $37.1 \%$ adopted total smoking ban within the home. Decreased likelihood of adopting total smoking bans was associated with current smoker status, low education attainment, lack of awareness on adverse health consequences of ETS, low level of support for tobacco control policies, and cohabitation with a smoker in both genders. Having smoke-free homes was also linked with age in women, place of residence and work smoking policy in indoor areas in men. Conclusions: Targeted activities to encourage adopting voluntary smoke-free rules among groups least likely to implement $100 \%$ smoking bans in the home and activities to decrease social acceptance of smoking in the presence of nonsmokers, children, pregnant woman are urgently needed.
\end{abstract}

Key words: Environmental tobacco smoke pollution, Environmental tobacco smoke, Smoke-free home, Tobacco control, Adults, Poland

\section{INTRODUCTION}

A vast number of studies revealed that exposure to environmental tobacco smoke (ETS) causes sickness, disability and death from a wide range of diseases [1].
Environmental tobacco smoke exposure causes serious negative health consequences, of which the increased risk of cardiovascular diseases, lung cancer, respiratory symptoms (for instance lower respiratory tract infections and

The work was financially supported by the Bloomberg Initiative to Reduce Tobacco Use, a program of Bloomberg Philanthropies. Project manager: Dorota Kaleta, M.P.H., Ph.D., coordinator of the Global Adult Tobacco Survey (GATS) in Poland.

Received: August 20, 2014. Accepted: April 13, 2015.

Corresponding author: D. Kaleta, Medical University of Lodz, Department of Preventive Medicine, Żeligowskiego 7/9, 90-752 Łódź, Poland (e-mail: dkaleta@op.pl). 
asthma) appear to be most important [2]. Environmental tobacco smoke exposure is also associated with poor pregnancy outcomes, including low birth weight [2]. Children exposed to secondhand smoke (SHS) are at an increased risk for sudden infant death syndrome (SIDS), acute respiratory infections, ear problems, and more severe asthma [3]. Smoking by parents causes respiratory symptoms and slows down lung growth in their children.

Exposure of adults to SHS has immediate adverse effects on the cardiovascular system and causes coronary heart disease and lung cancer [3]. It is estimated that ETS exposure contributes to about $1 \%$ of the total global disease burden, and represents about $10-15 \%$ of the disease burden caused by active smoking [1].

According to the World Health Organization (WHO), about $1 / 3$ of adults are regularly exposed to ETS [1]. In the European Union, 14\% of non-smokers are exposed to other people's tobacco smoke at home, and a 1/3 of working adults are exposed to ETS at the workplace at least some of the time [1]. According to WHO report, ETS causes about 600000 premature deaths per year worldwide [1]. In the European Union, ETS exposure at work is estimated to cause about 7600 deaths per year, while exposure at home results in 72100 deaths. In addition to adverse health consequences, ETS exposure also imposes economic burdens on individuals and countries, both the direct costs of health care and indirect costs from reduced productivity. Several studies estimate that $10 \%$ of total tobacco-related economic costs are attributable to secondhand tobacco smoke exposure [4].

In order to diminish ETS exposure, European Union countries, including Poland, focused mostly on implementing legal restrictions of smoking in public places and worksites. In recent years these policies were supported and carefully monitored. There is an ample evidence that smoke-free laws significantly reduce the level of exposure to ETS in work and public places [1]. However, even in countries where comprehensive and strict legislation has been enforced, many adults and children continue to be exposed to ETS in the home or other private settings. Conversely, not as much efforts were undertaken to encourage adoption of smoke-free rules in the private settings; this topic is far less explored and there is very little information on adoption of smoke-free home rules, especially in Poland.

To reduce this gap in national figures, we have evaluated prevalence and factors associated with voluntary implementation of smoking bans at home among economically active adult males and females in Poland.

\section{MATERIAL AND METHODS}

\section{Study design and sample}

Global Adult Tobacco Survey (GATS) was implemented in Poland between 2009 and 2010. It is the international project dedicated for systematically monitoring of use of tobacco products in adult population. Global Adult Tobacco Survey Poland is a nationally representative, household survey [5]. In Poland, the survey population selection process was based on multi-stage stratified geographically clustered sample of non-institutionalized population aged $\geq 15$ years, including men and women. A sample of 14000 households was randomly selected. Out of the 14000 households selected for the survey, 8948 (63.9\%) households and 7840 (93.9\%) sampled persons successfully completed the interviews. The total survey response rate was $65.1 \%$. Questionnaires were completed during face-to-face interviews at respondents' homes. More details on GATS methodology and overall approach are available in previously published papers.

\section{Study variables}

The individual GATS questionnaire is a wide-ranging tool covering the characteristics of the study participants and an extensive number of crucial information on tobacco use, including smoking tobacco products, SHS exposure, and many other aspects of tobacco epidemic. 
For the purpose of current analysis, smoker was defined as a person who smokes regularly, on daily basis or less frequently. Nonsmoker group comprised former smokers and never smokers.

Moreover, restrictions on smoking behavior at home were studied. To determine whether smoking was allowed in the respondent's home, smoking rules at home were recorded in the following categories: smoking is allowed, smoking is prohibited - with some exceptions from this rule, smoking is completely prohibited, and no rules. This question was asking about the rules for the inside spaces of the respondent's home. These include only enclosed areas of the home. Areas outside of the home, including patios, porches, etc. that are not fully enclosed were not taken into consideration.

Similarly, worksite smoking policies in indoor areas were assessed and subsequent categories were established: smoking is allowed everywhere, smoking is allowed in some indoor areas, smoking is prohibited in all indoor areas, no policy.

Furthermore, cohabitation with smoker(s) or nonsmoker(s) was considered, as well as living alone (living with non-smoker, living with smoker, living alone, or other). Awareness on adverse health consequences of ETS was examined as well. We classified our respondents as aware of the health consequences of ETS (those who answered "yes" to the question: "Do you think that tobacco ETS causes serious diseases?") and not aware (those who answered "no" and "do not know"). In addition, we evaluated support for tobacco control policies among study subjects (high, medium, low).

\section{Socio-demographic variables}

Data on gender and age of the respondents were included in our analysis as well. Moreover, the data on educational attainment of respondents were taken into consideration. Educational level was classified as: primary education, vocational education, secondary education, and high education. The measure of job characteristic classified subjects as white-collar workers (management or comanagement in a company or an enterprise, expert - independent professional with high qualifications and high education, administrative office staff in a company or an enterprise) and blue collar workers (trade or services employee foreman, technician supervising manual workers, skilled worker, non-skilled worker). We also determined whether respondents' place of residence was a rural or an urban area (urban area with $<50000,50$ 000-200 000, or $>200000$ inhabitants).

\section{Statistical analyses}

Statistical associations of the particular categories of characteristics in the analyzed subgroups were assessed with the $\mathrm{Chi}^{2}$ test. All analyses were performed in age groups: $15-29,30-39,40-49, \geq 50$ years and older. We used logistic regression analysis to evaluate associates of lack of adopting total smoking bans at home. Initially, univariate coefficients - odds ratios (OR) of the impact of odd variables on the lack of adopting total smoking bans within the home were calculated. This was followed by multifactorial analysis of the simultaneous effect of all statistically significant variables on lack of $100 \%$ smoke-free rules at home. For all analyses, $p<0.05$ was set as statistically significant. The calculation was performed by the statistical software package Statistica Windows XP version 8.0.

\section{RESULTS}

Out of the 14000 households selected for the survey, 8948 (63.9\%) households and 7840 (93.9\%) sampled subjects accomplished the interviews. The overall survey participation rate was $65.1 \%$. The data analyzed below covered 3696 economically active respondents, including 2108 men and 1588 women. Among this population, total smoke-free rules at home were adopted by $37.1 \%(\mathrm{~N}=1373)$ of study participants. The prevalence of total ban of smoking at home was declared by 
$37.4 \%$ male and $36.8 \%$ of female respondents. Of non-smokers, $51.5 \%(\mathrm{~N}=623)$ of males and $49.1 \%(\mathrm{~N}=530)$ females implemented total smoking ban in their place of living. Among smokers, $18.5 \%(\mathrm{~N}=165)$ males and $10.8 \%$ $(\mathrm{N}=55)$ females implemented $100 \%$ home smoke free rules. The prevalence of implementing smoking restrictions in the place of residence differ among smokers and nonsmokers by selected characteristics (Table 1 and 2).

It should be emphasized that smokers were less likely to implement smoke-free rules at their homes compared to non-smokers, and in all analyzed categories of variables, the percentage of smokers implementing total ban of smoking in their place of living was significantly lower than among non-smokers in both genders (Table 1 and 2). This is also true about comparisons of the prevalence of smoking ban that were made between smokers and nonsmokers cohabitating with smoking or not smoking spouses. It seems to be interesting that among male subjects, $17.9 \%$ of smokers have implemented smoke-free homes (SFH) if they lived with other smokers and at a similar level (18.7\%) among those living with non-smoking spouses. At the same time, over $80 \%$ of non-smokers implemented SFH in spite of the smoking status of the members of their household. In women, the prevalence of SFH among smokers was even lower and only $4.7 \%(\mathrm{~N}=4)$ of smokers living with non-smokers and $9.1 \%(\mathrm{~N}=4)$ of smokers living with other smokers adopted smoke-free rules at their place of residence.

\section{Logistic regression analysis}

\section{Univariate analysis}

Odds ratios (OR) and 95\% confidence intervals (CI) for lack of total smoking ban in the place of living and the number of variables: age, smoking status, place of residence, education, job classification, awareness on ETS health consequences, level of support for tobacco control polices, cohabitation with a smokers, smoking rules in indoor areas in the worksite were tested in a logistic regression model.
Table 2 and 3 shows results of the univariate and multivariate logistic regression analysis.

In the univariate logistic regression, decreased likelihood of adoption of complete smoking ban at home was associated with current smoker status, low education attainment, lack of awareness on adverse health consequences of ETS, low level of support for tobacco control policies, and cohabitation with a smoker. Job features were not associated with implementing smoke-free homes (SFH) either among males or females.

\section{Multivariate analysis}

The multivariate section confirmed the results obtained in the univariate study (Table 3). After adjusting for statistically significant variables, approximately 2 times lower likelihood of adopting smoking bans in the place of residence was still observed among male and female current smokers relative to non-smokers. Similarly, in respondents cohabiting with a smoker, at least doubled odds of lack of rules assuring total protection from ETS at home was recorded, compared with subjects living with non-smoker. Moreover, odds of lack of SFH was significantly higher among men with vocational education compared to those with high educational level. Among women, this association was strongest in the group with vocational education, but secondary education also over one-fold increased the likelihood of lack of SFH compared with highly educated subjects. However, shortage of knowledge on ETS health consequences in both genders was the strongest single predictor of lack of SFH. Those who were unaware of adverse health effects of ETS were approximately 3 times less likely to adopt total smoking ban at home compared to respondents perceiving ETS as dangerous to health (Table 3).

There was inverse association between level of support for tobacco control policies and likelihood of lack of implementation of SFH. Decreased support was associated with increased odds of lack of SFH, reaching close 
Table 1. Characteristics of male respondents including those implementing and not implementing complete smoking ban at home Global Adult Tobacco Survey Poland 2009-2010*

\begin{tabular}{|c|c|c|c|c|c|c|}
\hline \multirow{3}{*}{ Characteristic } & \multicolumn{6}{|c|}{$\begin{array}{c}\text { Males } \\
{[\mathrm{n}(\%)]}\end{array}$} \\
\hline & \multicolumn{2}{|c|}{ total } & \multicolumn{2}{|c|}{ smokers } & \multicolumn{2}{|c|}{ non-smokers } \\
\hline & ban & no ban & ban & no ban & ban & no ban \\
\hline Total & $788(37.4)$ & $1320(62.6)$ & $165(18.5)^{\mathrm{c}}$ & $725(81.5)^{c}$ & $623(51.5)$ & $595(48.5)$ \\
\hline \multicolumn{7}{|l|}{ Age [years] } \\
\hline $15-29$ & $173(37.9)$ & $283(62.1)$ & $42(22.0)^{\mathrm{c}}$ & $149(78.0)^{\mathrm{c}}$ & $131(49.4)$ & $134(50.6)$ \\
\hline $30-39$ & $278(41.9)$ & $385(58.1)$ & $68(24.5)^{\mathrm{c}}$ & $209(75.5)^{\mathrm{c}}$ & $210(54.4)$ & $176(45.6)$ \\
\hline $40-49$ & $179(33.1)$ & $361(66.9)$ & $36(14.7)^{c}$ & $208(85.3)^{c}$ & $143(48.3)$ & $153(51.7)$ \\
\hline$\geq 50$ & $159(35.3)$ & $291(64.7)$ & $19(10.7)^{\mathrm{c}}$ & $159(89.3)^{c}$ & $139(51.3)$ & $132(48.7)$ \\
\hline \multicolumn{7}{|l|}{ Place of residence } \\
\hline rural & $390(36.1)$ & $691(53.9)$ & $92(20.4)^{c}$ & $360(79.6)^{\mathrm{c}}$ & $298(47.4)$ & $331(52.6)$ \\
\hline \multicolumn{7}{|l|}{ urban } \\
\hline$<50000$ & $157(42.4)$ & $213(57.6)$ & $36(24.0)^{c}$ & $114(76.0)^{c}$ & $121(55.0)$ & $99(45.0)$ \\
\hline $50000-200000$ & $72(30.1)$ & $167(69.9)$ & $14(12.0)^{\mathrm{c}}$ & $103(88.0)^{c}$ & $58(47.5)$ & $64(52.5)$ \\
\hline$>200000$ & $169(40.3)$ & $249(59.7)$ & $23(13.4)^{c}$ & $148(86.6)^{c}$ & $146(59.1)$ & $101(40.9)$ \\
\hline \multicolumn{7}{|l|}{ Education } \\
\hline primary & $43(30.1)$ & $100(69.9)$ & $13(16.9)^{\mathrm{c}}$ & $64(83.1)^{c}$ & $30(45.5)$ & $36(54.5)$ \\
\hline vocational & $217(29.7)$ & $514(60.3)$ & $53(14.8)^{\mathrm{c}}$ & $304(85.2)^{\mathrm{c}}$ & $164(43.8)$ & $210(56.2)$ \\
\hline secondary & $347(40.0)$ & $520(60.0)$ & $74(21.6)^{c}$ & $268(88.4)^{c}$ & $273(52.0)$ & $252(48.0)$ \\
\hline high & $181(49.3)$ & $186(50.7)$ & $25(21.9)^{\mathrm{c}}$ & $89(78.1)^{c}$ & $156(61.7)$ & $97(38.3)$ \\
\hline \multicolumn{7}{|l|}{ Job classification } \\
\hline white collar workers & $337(45.1)$ & $410(54.9)$ & $70(25.2)^{\mathrm{c}}$ & $208(74.8)^{\mathrm{c}}$ & $267(56.9)$ & $202(43.1)$ \\
\hline blue collar workers & $451(33.1)$ & $910(66.9)$ & $95(15.5)^{\mathrm{c}}$ & $517(84.5)^{\mathrm{c}}$ & $356(47.5)$ & $393(52.5)$ \\
\hline \multicolumn{7}{|l|}{ Awareness on ETS health consequences } \\
\hline yes & $729(43.7)$ & $940(56.3)$ & $147(24.7)^{c}$ & $449(75.3)^{c}$ & $582(54.2)$ & $491(45.8)$ \\
\hline no & $59(13.4)$ & $380(86.6)$ & $18(6.1)^{\mathrm{c}}$ & $276(93.9)^{\mathrm{c}}$ & $41(26.5)$ & $104(73.5)$ \\
\hline \multicolumn{7}{|l|}{ Work smoking policy in indoor areas } \\
\hline smoking is allowed everywhere & $48(29.8)$ & $113(70.2)$ & $19(19.6)^{\mathrm{c}}$ & $78(80.4)^{c}$ & $29(45.3)$ & $35(54.7)$ \\
\hline smoking is allowed in some indoor areas & $261(32.1)$ & $551(67.9)$ & $55(15.1)^{\mathrm{c}}$ & $310(84.9)^{c}$ & $206(46.1)$ & $241(53.9)$ \\
\hline smoking is prohibited in all indoor areas & $337(49.6)$ & $342(50.4)$ & $56(24.1)^{c}$ & $176(75.9)^{\mathrm{c}}$ & $281(62.7)$ & $166(37.3)$ \\
\hline no policy & $35(32.1)$ & $74(67.9)$ & $10(19.6)^{b}$ & $41(80.4)^{b}$ & $25(43.1)$ & $33(56.9)$ \\
\hline \multicolumn{7}{|l|}{ Support for tobacco control } \\
\hline high & $618(48.4)$ & $658(51.6)$ & $94(26.4)^{c}$ & $262(73.9)^{c}$ & $524(57.0)$ & $396(43.0)$ \\
\hline medium & $137(23.6)$ & $444(76.4)$ & $58(16.5)^{c}$ & $294(84.5)^{c}$ & $79(34.5)$ & $150(64.5)$ \\
\hline low & $33(13.1)$ & $218(86.9)$ & $13(7.1)^{\mathrm{c}}$ & $169(92.9)^{c}$ & $20(29.0)$ & $49(71.0)$ \\
\hline
\end{tabular}


Table 1. Characteristics of male respondents including those implementing and not implementing complete smoking ban at home Global Adult Tobacco Survey Poland 2009-2010* - cont.

\begin{tabular}{lrrrrrr}
\hline \multirow{2}{*}{ Characteristic } & \multicolumn{7}{c}{$\begin{array}{c}\text { Males } \\
{[\mathrm{n}(\%)]}\end{array}$} \\
\cline { 2 - 7 } & \multicolumn{9}{c}{ total } & \multicolumn{2}{c}{ smokers } & \multicolumn{2}{c}{ non-smokers } \\
\cline { 2 - 7 } & \multicolumn{1}{c}{ ban } & no ban & ban & no ban & ban & no ban \\
\hline Cohabitation with smoker(s) & & & & & & \\
no & $132(38.0)$ & $215(62.0)$ & $14(18.7)^{c}$ & $61(91.3)^{c}$ & $118(43.4)$ & $154(56.6)$ \\
yes & $40(20.2)$ & $158(79.8)$ & $26(17.9)^{\mathrm{a}}$ & $119(82.1)^{\mathrm{a}}$ & $14(32.6)$ & $39(67.4)$ \\
living alone or other & $616(39.4)$ & $947(60.6)$ & $125(18.7)^{\mathrm{c}}$ & $545(81.3)^{\mathrm{c}}$ & $491(55.0)$ & $402(67.5)$ \\
\hline
\end{tabular}

ETS - environmental tobacco smoke.

* Some strata do not sum up to initial total value because of missing data.

Smokers vs. non-smokers: ${ }^{\mathrm{a}} \mathrm{p} \leq 0.05 ;{ }^{\mathrm{b}} \mathrm{p} \leq 0.01 ;{ }^{\mathrm{c}} \mathrm{p} \leq 0.001$.

Table 2. Characteristics of female respondents including those implementing and not implementing complete smoking ban at home Global Adult Tobacco Survey Poland 2009-2010*

\begin{tabular}{|c|c|c|c|c|c|c|}
\hline \multirow{3}{*}{ Characteristic } & \multicolumn{6}{|c|}{$\begin{array}{l}\text { Females } \\
{[\mathrm{n}(\%)]}\end{array}$} \\
\hline & \multicolumn{2}{|c|}{ total } & \multicolumn{2}{|c|}{ smokers } & \multicolumn{2}{|c|}{ non-smokers } \\
\hline & ban & no ban & ban & no ban & ban & no ban \\
\hline Total & $585(36.8)$ & $1003(73.2)$ & $55(10.8)^{\mathrm{c}}$ & $453(89.2)^{c}$ & $530(49.1)$ & $550(50.9)$ \\
\hline \multicolumn{7}{|l|}{ Age [years] } \\
\hline $15-29$ & $113(35.6)$ & $204(64.4)$ & $5(5.4)^{\mathrm{c}}$ & $88(94.6)^{c}$ & $108(48.2)$ & $116(51.8)$ \\
\hline $30-39$ & $228(45.2)$ & $276(54.8)$ & $25(18.9)^{\mathrm{c}}$ & $107(81.1)^{c}$ & $203(54.6)$ & $169(45.4)$ \\
\hline $40-49$ & $152(34.5)$ & $288(65.5)$ & $18(11.3)^{\mathrm{c}}$ & $142(88.7)^{\mathrm{c}}$ & $134(47.9)$ & $146(52.1)$ \\
\hline$\geq 50$ & $92(29.0)$ & $225(71.0)$ & $7(5.7)^{\mathrm{c}}$ & $116(94.3)^{c}$ & $85(41.7)$ & $119(58.3)$ \\
\hline \multicolumn{7}{|l|}{ Place of residence } \\
\hline rural & $242(36.2)$ & $427(73.8)$ & $21(12.2)^{\mathrm{c}}$ & $151(87.8)^{c}$ & $221(44.5)$ & $276(55.5)$ \\
\hline \multicolumn{7}{|l|}{ urban } \\
\hline$<50000$ & $109(39.8)$ & $165(60.2)$ & $15(17.0)^{\mathrm{c}}$ & $73(83.0)^{\mathrm{c}}$ & $94(50.5)$ & $92(49.5)$ \\
\hline $50000-200000$ & $82(33.6)$ & $162(66.4)$ & $5(5.8)^{\mathrm{c}}$ & $81(84.2)^{\mathrm{c}}$ & $77(48.7)$ & $81(51.3)$ \\
\hline$>200000$ & $152(37.9)$ & 249 (62.1) & $14(8.6)^{\mathrm{c}}$ & $148(91.4)^{c}$ & $138(57.7)$ & $101(42.3)$ \\
\hline \multicolumn{7}{|l|}{ Education } \\
\hline primary & $16(21.9)$ & $57(78.1)$ & $4(11.1)^{a}$ & $32(88.9)^{\mathrm{a}}$ & $12(32.0)$ & $25(68.0)$ \\
\hline vocational & $77(26.3)$ & $216(73.7)$ & $6(5.4)^{\mathrm{c}}$ & $105(94.6)^{c}$ & $71(39.0)$ & $111(61.0)$ \\
\hline secondary & $271(35.0)$ & $504(65.0)$ & $26(10.2)^{c}$ & $230(89.8)^{c}$ & $245(47.2)$ & $274(52.8)$ \\
\hline high & $221(49.4)$ & $226(50.6)$ & $19(18.1)^{\mathrm{c}}$ & $86(81.9)^{\mathrm{c}}$ & $202(59.1)$ & $140(40.9)$ \\
\hline \multicolumn{7}{|l|}{ Job classification } \\
\hline white collar workers & $358(42.4)$ & $487(57.6)$ & $36(14.1)^{c}$ & $220(85.9)^{c}$ & $322(54.7)$ & $267(55.3)$ \\
\hline blue collar workers & $227(30.5)$ & $516(69.5)$ & $19(7.5)^{\mathrm{c}}$ & $233(92.5)^{\mathrm{c}}$ & $208(42.4)$ & $283(57.6)$ \\
\hline
\end{tabular}


Table 2. Characteristics of female respondents including those implementing and not implementing complete smoking ban at home Global Adult Tobacco Survey Poland 2009-2010* - cont.

\begin{tabular}{|c|c|c|c|c|c|c|}
\hline \multirow{3}{*}{ Characteristic } & \multicolumn{6}{|c|}{$\begin{array}{l}\text { Females } \\
{[\mathrm{n}(\%)]}\end{array}$} \\
\hline & \multicolumn{2}{|c|}{ total } & \multicolumn{2}{|c|}{ smokers } & \multicolumn{2}{|c|}{ non-smokers } \\
\hline & ban & no ban & ban & no ban & ban & no ban \\
\hline \multicolumn{7}{|l|}{ Awareness on ETS health consequences } \\
\hline yes & $567(41.4)$ & $803(58.6)$ & $51(14.0)^{\mathrm{c}}$ & $313(86.0)^{c}$ & $516(51.3)$ & $490(48.7)$ \\
\hline no & $18(8.3)$ & $200(91.7)$ & $4(2.8)^{\mathrm{c}}$ & $140(97.2)^{c}$ & $14(18.9)$ & $60(81.1)$ \\
\hline \multicolumn{7}{|l|}{ Workplace smoking policy in indoor areas } \\
\hline smoking is allowed everywhere & $9(21.9)$ & $32(78.1)$ & $1(4.2)^{b}$ & $23(95.8)^{b}$ & $8(47.1)$ & $9(52.9)$ \\
\hline smoking is allowed in some indoor areas & $171(31.1)$ & $378(68.9)$ & $21(9.1)^{\mathrm{c}}$ & $211(90.9)^{c}$ & $150(47.3)$ & $167(52.7)$ \\
\hline smoking is prohibited in all indoor areas & $338(44.7)$ & $418(55.3)$ & $29(14.9)^{\mathrm{c}}$ & $165(85.1)^{c}$ & $309(55.0)$ & $253(45.0)$ \\
\hline no policy & $10(37.0)$ & $17(63.0)$ & $0(0.0)^{\mathrm{a}}$ & $8(100.0)^{\mathrm{a}}$ & $10(52.6)$ & $9(47.4)$ \\
\hline \multicolumn{7}{|l|}{ Support for tobacco control } \\
\hline \multicolumn{7}{|l|}{ high } \\
\hline medium & $490(46.2)$ & $570(53.8)$ & $35(17.2)^{c}$ & $168(82.8)^{\mathrm{c}}$ & $455(53.1)$ & $402(46.9)$ \\
\hline low & $76(19.6)$ & $311(80.4)$ & $16(7.3)^{c}$ & $204(92.7)^{c}$ & $60(35.9)$ & $107(64.1)$ \\
\hline \multicolumn{7}{|l|}{ Cohabitation with smoker(s) } \\
\hline no & $19(13.5)$ & $122(86.5)$ & $4(4.7)^{c}$ & $81(95.3)^{c}$ & $15(26.8)$ & $41(73.2)$ \\
\hline yes & $137(39.8)$ & $207(60.2)$ & $4(9.1)^{c}$ & $40(90.9)^{c}$ & $133(44.3)$ & $167(55.7)$ \\
\hline living alone or other & $14(13.7)$ & $88(86.3)$ & $10(12.8)$ & $68(87.2)$ & $4(16.7)$ & $20(83.3)$ \\
\hline
\end{tabular}

Abbreviations as in Table 1.

Table 3. Odds ratios (OR) and 95\% confidence intervals $(\mathrm{CI})$ for lack of implementation of complete smoke-free homes rules vs. selected socio-demographic characteristics - Global Adult Tobacco Survey Poland 2009-2010

\begin{tabular}{|c|c|c|c|c|c|c|c|c|}
\hline \multirow{3}{*}{ Characteristic } & \multicolumn{4}{|c|}{$\begin{array}{c}\text { Males } \\
(\mathrm{N}=1320)\end{array}$} & \multicolumn{4}{|c|}{$\begin{array}{c}\text { Females } \\
(\mathrm{N}=1003)\end{array}$} \\
\hline & \multicolumn{2}{|c|}{$\begin{array}{l}\text { univariate logistic } \\
\text { regression }\end{array}$} & \multicolumn{2}{|c|}{$\begin{array}{l}\text { multivariate logistic } \\
\text { regression }^{\mathrm{a}}\end{array}$} & \multicolumn{2}{|c|}{$\begin{array}{l}\text { univariate logistic } \\
\text { regression }\end{array}$} & \multicolumn{2}{|c|}{$\begin{array}{c}\text { multivariate logistic } \\
\text { regression }^{\mathrm{a}}\end{array}$} \\
\hline & OR & $95 \% \mathrm{CI}$ & OR & $95 \% \mathrm{CI}$ & OR & $95 \% \mathrm{CI}$ & OR & $95 \% \mathrm{CI}$ \\
\hline \multicolumn{9}{|l|}{ Age [years] } \\
\hline $15-29$ & 1.00 & reference & & & 1.00 & reference & 1.00 & reference \\
\hline $30-39$ & 0.85 & $0.66-1.08$ & & & 0.67 & $0.50-0.89^{c}$ & 0.61 & $0.43-0.87^{\mathrm{c}}$ \\
\hline $40-49$ & 1.23 & $0.95-1.60$ & & & 1.05 & $0.78-1.42$ & 0.84 & $0.58-1.22$ \\
\hline$\geq 50$ & 1.13 & $0.86-1.48$ & & & 1.41 & $1.01-1.97^{\mathrm{b}}$ & 1.11 & $0.74-1.68$ \\
\hline \multicolumn{9}{|l|}{ Smoking status } \\
\hline smoker & 4.60 & $3.75-5.64^{d}$ & 1.99 & $1.34-2.95^{\mathrm{d}}$ & 7.94 & $5.85-10.77^{\mathrm{d}}$ & 2.59 & $1.52-4.41^{\mathrm{d}}$ \\
\hline non-smoker & 1.00 & reference & 1.00 & reference & 1.00 & reference & 1.00 & reference \\
\hline
\end{tabular}


Table 3. Odds ratios (OR) and $95 \%$ confidence intervals (CI) for lack of implementation of complete smoke-free homes rules vs. selected socio-demographic characteristics - Global Adult Tobacco Survey Poland 2009-2010 - cont.

\begin{tabular}{|c|c|c|c|c|c|c|c|c|}
\hline \multirow{3}{*}{ Characteristic } & \multicolumn{4}{|c|}{$\begin{array}{c}\text { Males } \\
(\mathrm{N}=1320)\end{array}$} & \multicolumn{4}{|c|}{$\begin{array}{c}\text { Females } \\
(\mathrm{N}=1003)\end{array}$} \\
\hline & \multicolumn{2}{|c|}{$\begin{array}{l}\text { univariate logistic } \\
\text { regression }\end{array}$} & \multicolumn{2}{|c|}{$\begin{array}{l}\text { multivariate logistic } \\
\text { regression }^{\mathrm{a}}\end{array}$} & \multicolumn{2}{|c|}{$\begin{array}{l}\text { univariate logistic } \\
\text { regression }\end{array}$} & \multicolumn{2}{|c|}{$\begin{array}{l}\text { multivariate logistic } \\
\text { regression }\end{array}$} \\
\hline & OR & $95 \% \mathrm{CI}$ & OR & $95 \% \mathrm{CI}$ & OR & $95 \% \mathrm{CI}$ & OR & $95 \% \mathrm{CI}$ \\
\hline \multicolumn{9}{|l|}{ Place of residence } \\
\hline rural & 1.20 & $0.95-1.52$ & 1.17 & $0.88-1.57$ & 1.08 & $0.83-1.39$ & & \\
\hline \multicolumn{9}{|l|}{ urban } \\
\hline$<50000$ & 0.92 & $0.69-1.22$ & 1.02 & $0.72-1.43$ & 0.92 & $0.67-1.27$ & & \\
\hline 50 000-200 000 & 1.57 & $1.12-2.21^{\mathrm{c}}$ & 1.70 & $1.15-2.54^{\mathrm{c}}$ & 1.21 & $0.86-1.68$ & & \\
\hline$>200000$ & 1.00 & reference & 1.00 & reference & 1.00 & reference & 1.00 & reference \\
\hline \multicolumn{9}{|l|}{ Education } \\
\hline primary & 2.26 & $1.51-3.40^{\mathrm{d}}$ & 1.49 & $0.85-2.58$ & 3.48 & $1.94-6.25^{\mathrm{d}}$ & 1.21 & $0.53-2.80$ \\
\hline vocational & 2.30 & $1.80-2.96^{\mathrm{d}}$ & 1.79 & $1.31-2.45^{\mathrm{d}}$ & 2.74 & $2.00-3.77^{\mathrm{d}}$ & 2.04 & $1.37-3.05^{\mathrm{d}}$ \\
\hline secondary & 1.46 & $1.15-1.85^{\mathrm{d}}$ & 1.18 & $0.88-1.57$ & 1.82 & $1.44-2.30^{\mathrm{d}}$ & 1.41 & $1.06-1.86^{\mathrm{b}}$ \\
\hline high & 1.00 & reference & 1.00 & reference & 1.00 & reference & 1.00 & reference \\
\hline \multicolumn{9}{|l|}{ Job classification } \\
\hline white collar workers & 1.00 & reference & & & 1.00 & reference & & \\
\hline blue collar workers & 1.18 & $0.94-1.47$ & & & 0.81 & $0.57-1.15$ & & \\
\hline \multicolumn{9}{|l|}{ Awareness on ETS health consequences } \\
\hline yes & 1.00 & reference & 1.00 & reference & 1.00 & reference & 1.00 & reference \\
\hline no & 4.99 & $3.73-6.68^{\mathrm{d}}$ & 2.63 & $1.88-3.67^{\mathrm{d}}$ & 7.85 & $4.78-12.86^{\mathrm{d}}$ & 2.97 & $1.72-5.14^{\mathrm{d}}$ \\
\hline \multicolumn{9}{|l|}{ Work smoking policy in indoor areas } \\
\hline smoking is allowed everywhere & 2.32 & $1.61-3.33^{\mathrm{d}}$ & 1.18 & $0.77-1.81$ & 2.88 & $1.35-6.11^{\mathrm{c}}$ & 1.52 & $0.63-3.68$ \\
\hline smoking is allowed in some indoor areas & 2.08 & $1.71-2.54^{\mathrm{d}}$ & 1.66 & $1.31-2.10^{\mathrm{d}}$ & 1.78 & $1.42-2.25^{\mathrm{d}}$ & 1.23 & $0.94-1.60$ \\
\hline smoking is prohibited in all indoor areas & 1.00 & reference & 1.00 & reference & 1.00 & reference & 1.00 & reference \\
\hline no policy & 2.08 & $1.36-3.18^{\mathrm{d}}$ & 1.59 & $0.99-2.57$ & 1.37 & $0.62-3.04$ & 1.27 & $0.52-3.06$ \\
\hline \multicolumn{9}{|l|}{ Support for tobacco control policies } \\
\hline high & 1.00 & reference & 1.00 & reference & 1.00 & reference & 1.00 & reference \\
\hline medium & 3.26 & $2.63-4.04^{\mathrm{d}}$ & 1.77 & $1.36-2.29^{\mathrm{d}}$ & 3.61 & $2.75-4.72^{\mathrm{d}}$ & 1.93 & $1.39-2.68^{\mathrm{d}}$ \\
\hline low & 6.32 & $3.98-10.02^{\mathrm{d}}$ & 2.71 & $1.59-4.60^{\mathrm{d}}$ & 5.89 & $3.25-10.67^{\mathrm{d}}$ & 2.70 & $1.35-5.39^{c}$ \\
\hline \multicolumn{9}{|l|}{ Cohabitation with smoker(s) } \\
\hline no & 1.00 & reference & 1.00 & reference & 1.00 & reference & 1.00 & reference \\
\hline yes & 4.26 & $3.49-5.20^{\mathrm{d}}$ & 1.83 & $1.24-2.69^{c}$ & 7.38 & $5.44-10.01^{\mathrm{d}}$ & 2.50 & $1.49-4.20^{\mathrm{d}}$ \\
\hline living alone or other & 3.37 & $2.18-5.22^{\mathrm{d}}$ & 2.08 & $1.25-3.49^{c}$ & 2.16 & $1.36-3.42^{\mathrm{d}}$ & 1.51 & $0.90-2.53$ \\
\hline
\end{tabular}

ETS - environmental tobacco smoke.

${ }^{\text {a }}$ Fully adjusted model including all statistically significant characteristics.

${ }^{\mathrm{b}} \mathrm{p} \leq 0.05$.

${ }^{c} \mathrm{p} \leq 0.01$.

${ }^{\mathrm{d}} \mathrm{p} \leq 0.001$. 
to 3-fold rate among subjects declaring low level of support related to those with high level of support for tobacco control policies.

Furthermore, higher likelihood of SFH was still observed among the females aged 30-39 compared with those aged 15-29. Among men, also place of residence and workplace policies on indoor smoking were associated with SFH adoption.

Job classification was not associated with home smoking restrictions in both genders.

\section{DISCUSSION}

In our study, we noticed low prevalence of $100 \%$ smokefree homes that were adopted only by $37.1 \%$ (50.2\% among nonsmokers, $15.7 \%$ among smokers, respectively) of study participants, compared to $81.1 \%$ (89.1\% among nonsmokers and $48 \%$ among smokers, respectively) in the USA [6]. It was also lower than in other European countries; for instance a complete home smoking ban was reported by $59.5 \%$ of French, $63.5 \%$ of Irish, $61.3 \%$ of Italian, $74.4 \%$ of Czech and $87 \%$ of Swedish females in 2010 [7]. Considering data from all those European countries together, the prevalence of a smoking ban at home varied between smokers and nonsmokers [7].

It was reported among $75 \%$ of the nonsmokers and 50\% smokers. Differences across countries were more apparent among smokers than among nonsmokers. Sweden had the largest proportion of participants who reported having a smoke-free home, and this was the case both among smokers and nonsmokers [7]. Among nonsmoking participants, Italy had the lowest proportion with a home smoking restriction (66\%), while among smokers, France had the lowest proportion having a smoking restriction at home (31\%). Among females employed outside of the home, Ireland had the lowest proportion of participants reporting that smoking was allowed in their immediate working area, while in Italy the proportion was highest.
Findings form GATS indicate that most adults are not protected by complete voluntary smoke-free rules in their homes. Data from Poland are comparable with previously reported estimates for 1992-1993 (43.1\%) from the United States [6]. This reflects previous lower interest in Poland in implementing voluntary smoking bans in private places and low number of activities undertaken to solve this problem compared to other countries.

Moreover, there are differences among subpopulations in adopting $100 \% \mathrm{SFH}$, and some groups are at increased risk of lack of effective protection within their homes. The prevalence of smoke-free rules was generally lower among individuals with lower education and smokers. Besides, in Poland, lack of awareness on adverse health consequences of ETS, low level of support for tobacco control policies, and cohabitation with a smoker was associated with lack of protection from tobacco pollution at home in both genders. Similarly Heck et al. found that having a home smoking ban was related to smoking behavior, demographics, beliefs and personal preference. Gonzales et al. in the study of prevalence and predictors of home and automobile smoking bans among US and Mexico-born Hispanic women with young children reveled that current smoking and presence of other adult smokers in the home were associated with significantly increased odds of not having a complete home or automobile smoking ban [8]. Our results are also supported by findings from USA, where socio-economic disparities in SFH adoption exist and in general are associated with lower socio-economic status. As King et al. suggested, these findings may be due to the higher rates of cigarette smoking among disadvantaged groups compared to more affluent respondents, cultural factors related to the social disapproval of smoking, or differences in receptivity toward tobacco-related health messages and understanding of the health hazards associated with SHS exposure [6]. Our results are also in line with a study on SHS exposure among adults by King et al., who showed that among all respondents, exposure to SHS in the home 
was $54.9 \%$ in Bangladesh, $27.9 \%$ in Brazil, $67.3 \%$ in China, $62.5 \%$ in Egypt, $40 \%$ in India, $17.3 \%$ in Mexico, $54.4 \%$ in the Philippines, $34.7 \%$ in Russia, $33.2 \%$ in Thailand, $56.3 \%$ in Turkey, $23.5 \%$ in Ukraine, $34 \%$ in Uruguay, and $73.1 \%$ in Vietnam [9]. Thus, Poland belongs to the countries with medium to high prevalence of SHS exposure among low/medium income countries. But compared to high income countries, for instance United States, prevalence of residential SHS in Poland was close to 10 times higher (59\%) than among US citizens (6\%) [9].

This reflects low prevalence of smoking bans at home in Poland and probably differences in comprehensiveness of tobacco control measures implemented in those countries and social approval for smoking. Another aspect could be implementation of country-specific interventions and polices with special focus on smoke-free public places and adopting 100\% smoke-free homes, which are uncommon in Poland.

Smoking bans are mainly instituted to protect nonsmokers and to decrease overall exposure to SHS [10]. However, Zhu et al. in his review showed that the bans also help smokers quit smoking and prevent relapse among former smokers [10]. Some studies showed that as workplaces implement such policies, people adopt similar policies at home, and effects of smoke-free homes on cessation are even more consistent than those of smoke-free worksites. This is partly because these data are correlational. Worksite policies are imposed, while home bans may reflect smokers' own motivation to quit [10].

Likewise, GATS study in Poland also showed higher likelihood for adoption of SFH among men who have smoking bans at work. Although SFH has not yet become a focus of public health experts, and wide-ranging campaigns to encourage the introduction of smoking restrictions in the home have not been undertaken in Poland, reports from other countries with more experience are quite optimistic. First experiences from countries with longer-than-in-Poland history of tobacco control, more intensive activities and comprehensive laws in this area show that positive effects of undertaken activities appear after some time. In Australia, for example, the percentages of visitors being discouraged from smoking inside the home rose from $27 \%$ in 1989 to $53 \%$ in 1997 . The number of smokers who reported always smoking outside the home rose from $20 \%$ in 1995 to $28 \%$ in 1997 . Not smoking in the presence of children rose from $14 \%$ in 1989 to $33 \%$ in 1996 [11]. Other findings demonstrate that smoke-free legislation may stimulate smokers and nonsmokers to establish total smoking bans in their homes and help improve regulations on smoking [12-14]. Finally, there is a number of examples of effective tobacco control programs that can be successfully adopted in our country.

\section{Study limitations and strengths}

Global Adult Tobacco Survey is a cross-sectional study based on the questionnaire, what can lead to recall bias. However, questionnaires seem to be cost-efficient, easy to collect, allow approaching high number of respondents, and have been found to be valid tools in most epidemiological studies of large populations. Moreover, due to low number of respondents in several cells, we were unable to run separate logistic regression models to analyze odds for lack of SFH rules in smokers and non-smokers. Furthermore, we do not have the information whether smoking policies in the home are enforced. The cross-sectional nature of this study also limits the ability to conclude about what is the cause and what is the effect in the observed correlation.

Strengths of this study include determining the smoking restrictions applied to anyone inside the respondent's home, for example visitors (people from outside of the interviewed household). Moreover, GATS is a countrywide, representative household survey of adults $\geq 15$ years of age using a standard core survey, sample design, and data collection and management procedures that have been revised and approved by international experts. To the best 
of our knowledge, GATS has delivered probably the most reliable and the most recent figures on SFH implementation in Poland.

\section{CONCLUSIONS}

Exposure to secondhand smoke remains a serious public health problem in Poland. Data from GATS suggest that the home is a very important target and least utilized opportunity for reducing exposures to secondhand smoke among Poles. Currently in Poland, prevalence of SFH is low and especially too many smokers continue to cause their families to be exposed to tobacco smoke. It also indicates the common tolerance of smoking and exposure of other people to secondhand smoke.

Actions initiating move towards adopting SFH are needed. Substantial support is necessary to encourage and provide the conditions under which more homes can be smokefree [14]. There is a need to employ strategies that influence smoking practices of all household members. Experiences from other countries suggest that progress can be achieved in ETS control and SFH adoption through activities at a population level [11]. Efforts to make smoking and ETS exposure socially unacceptable should be continued. Considering this, disseminating the information on the risks of exposure to environmental tobacco smoke at home, putting into practice smoke-free home rules is of significant public health importance [15].

Educational activities, as well as local or national mass media campaigns are necessary to raise awareness of the adverse health effects of smoking. An important component of elevating awareness of the harmfulness of tobacco smoke are also text and pictorial warnings on cigarette packs. Moreover, strong clean indoor air laws are associated with large increases in voluntary smoke-free home policies both in the homes with and without smokers [13]. The "norm of spreading clean indoor air laws" suggests the need for further strengthening the legislations intended to make public places and worksites smoke-free [16]. Additionally, interventions to encourage the adoption of smoke-free policies by owners and managers of multiunit housing should be undertaken [17]. Smoke-free homes should be promoted in our country also to help smokers quit smoking, in spite of limited cessation services [18]. In Poland, there is also a need for further surveys to explain determinants of voluntary adopting smoke-free rules at homes.

\section{ACKNOWLEDGMENTS}

We would like to thank the representatives of the Global Adult Tobacco Survey Scientific Committee (Poland) including: Professor Bolesław Samoliński (Medical University, Warszawa), Professor Witold Zatoński (Cancer Center and Institute of Oncology, Warszawa), Dr. Przemysław Biliński (Chief Sanitary Inspector, Warszawa), as well as members of the Technical and Survey Staff Team, and our partners from the World Health Organization (Dr. Lubna Bhatti, Dr. Sameer Pujari), Centers for Disease Control and Prevention (Dr. Samira Asma, Dr. Krishna Mohan Palipudi), CDC Foundation, Johns Hopkins Bloomberg School of Public Health and the RTI International for their contribution and support. The list of the Study Collaborative Group is available at http://www.mz.gov.pl.

\section{REFERENCES}

1. World Health Organization. WHO report on the global tobacco epidemic. Implementing smoke-free environments. Geneva: The Organization; 2009.

2. World Health Organization, International Agency for Research on Cancer. Tobacco smoke and involuntary smoking. Lyon: WHO, IARC; 2004.

3. U.S. Department of Health and Human Services. Children and secondhand smoke exposure. Excerpts from the health consequences of involuntary exposure to tobacco smoke: A report of the surgeon general. Atlanta (GA): U.S. Department of Health and Human Services, Centers for Disease Control and Prevention, Coordinating Center for Health Promotion, National Center for Chronic Disease Prevention and Health Promotion, Office on Smoking and Health; 2007. 
4. The Adopting Standards and Specifications for Educational Content (ASPECT) Consortium. Tobacco or health in the European Union past, present and future. Luxembourg: Office for Official Publications of the European Communities; 2004.

5. Włodarczyk A, Raciborski F, Opoczyńska D, Samoliński B, GATS PWG. Daily tobacco smoking patterns in rural and urban areas of Poland - The results of the GATS study. Ann Agric Environ Med. 2013 Sep 20;20(3):588-94.

6. King BA, Dube SR, Homa DM. Smoke-free rules and secondhand smoke exposure in homes and vehicles among US adults, 2009-2010. Prev Chronic Dis. 2013;10:120218, http://dx.doi.org/10.5888/pcd10.120218.

7. Heck JE, Stucker I, Allwright S, Gritz ER, Haglunde M, Healton CG, et al. Home and workplace smoking bans in Italy, Ireland, Sweden, France and the Czech Republic. Eur Respir J. 2010;35:969-79, http://dx.doi. org/10.1183/09031936.00066809.

8. Gonzales M, Malcoe LH, Kegler MC, Espinoza J. Prevalence and predictors of home and automobile smoking bans and child environmental tobacco smoke exposure: A crosssectional study of U.S.- and Mexico-born Hispanic women with young children. BMC Public Health. 2006;6:265, http:// dx.doi.org/10.1186/1471-2458-6-265.

9. King BA, Mirza SA, Babb SD; GATS Collaborating Group. A cross-country comparison of secondhand smoke exposure among adults: Findings from the Global Adult Tobacco Survey (GATS). Tob Control. 2013 Jul;22(4):e5, http://dx.doi. org/10.1136/tobaccocontrol-2012-050582. Erratum in: Tob Control. 2013 Sep;22(5):362, http://dx.doi.org/10.1136/tobaccocontrol-2012-050582corr1.

10. Zhu SH, Lee M, Zhuang YL, Gamst A, Wolfson T. Interventions to increase smoking cessation at the population level: How much progress has been made in the last 2 decades? Tob Control. 2012;21:110-8, http://dx.doi.org/10.1136/tobaccocontrol-2011-050371.
11. Borland R, Mullins R, Trotter L, White V. Trends in environmental tobacco smoke restrictions in the home in Victoria, Australia. Tob Control. 1999;8:266-71, http://dx.doi. org/10.1136/tc.8.3.266.

12. Mons U, Nagelhout GE, Allwright S, Guignard R, van den Putte B, Willemsen MC, et al. Impact of national smoke-free legislation on home smoking bans: Findings from the International Tobacco Control Policy Evaluation Project Europe Surveys. Tob Control. 2013;22:e2-9, http:// dx.doi.org/10.1136/tobaccocontrol-2011-050131.

13. Cheng KW, Glantz SA, Lightwood JM. Association between smokefree laws and voluntary smokefree-home rules. Am J Prev Med. 2011;41(6):566-72, http://dx.doi. org/10.1016/j.amepre.2011.08.014.

14. Borland R, Yong H-H, Cummings KM, Hyland A, Anderson S, Fong GT. Determinants and consequences of smokefree homes: Findings from the International Tobacco Control (ITC) Four Country Survey. Tob Control. 2006;15(Suppl III):iii42-50, http://dx.doi.org/10.1136/tc.2005.012492.

15. World Health Organization. WHO report on the global tobacco epidemic, 2011: Warning about the dangers of tobacco. Geneva: WHO; 2011.

16. World Health Organization. WHO Report on the global tobacco epidemic, 2008; The MPOWER package. Geneva: WHO; 2008.

17. King BA, Mahoney MC, Cummings KM, Hyland AJ. Intervention to promote smoke-free policies among multiunit housing operators. J Public Health Manag Pract. 2011 May-Jun;17(3):E1-8, http://dx.doi.org/10.1097/PHH. 0b013e3181ffd8e3.

18. Hyland A, Higbee C, Travers MJ, van Deusen A, BansalTravers M, King B, et al. Smoke-free homes and smoking cessation and relapse in a longitudinal population of adults. Nicotine Tob Res. 2009;11:614-8, http://dx.doi.org/10.1093/ ntr/ntp022.

This work is available in Open Access model and licensed under a Creative Commons Attribution-NonCommercial 3.0 Poland License - http://creativecommons.org/ licenses/by-nc/3.0/pl/deed.en. 